\title{
a-Blocker resolves VUR caused by bladder neck dysfunction
}

The failure of conventional therapies for vesicoureteral reflux (VUR) might be due to underdiagnosis of the underlying cause; primary bladder neck dysfunction (PBND), for example, is often overlooked in children. In a recent study from Iran, Abdol-Mohammad Kajbafzadeh and colleagues report the successful treatment of VUR secondary to PBND with prazosin-an $\alpha$-blocker.

The researchers performed a doubleblind randomized controlled trial of 62 children (mean age 7.9 years) with proven VUR and PBND. Patients received either $0.025 \mathrm{mg} / \mathrm{kg}$ prazosin $(n=40)$ or placebo $(n=22)$ nightly for a week, increasing to $0.5 \mathrm{mg}$ twice daily thereafter.

After 6 months, a 40\% reduction in reflux grade (assessed using The International Reflux Study in Children classification) was reported in the prazosin group, compared to a $11 \%$ decrease in patients who received placebo $(P=0.016)$. This favorable outcome persisted at 12 months, when prazosin was associated with a $60 \%$ decrease in reflux grade, compared to $17 \%$ in the placebo cohort $(P=0.002)$. Moreover, significant improvement in bladder neck opening time was observed in the prazosin group; a 58\% decrease at 12 months, compared to only $18 \%$ in the placebo group. Similarly, average flow rate, $Q_{\max }$, postvoid residual volume, bladder compliance and mean maximal detrusor pressure also improved significantly in the treatment group compared to the control arm, at both 6-month and 12-month follow-up.

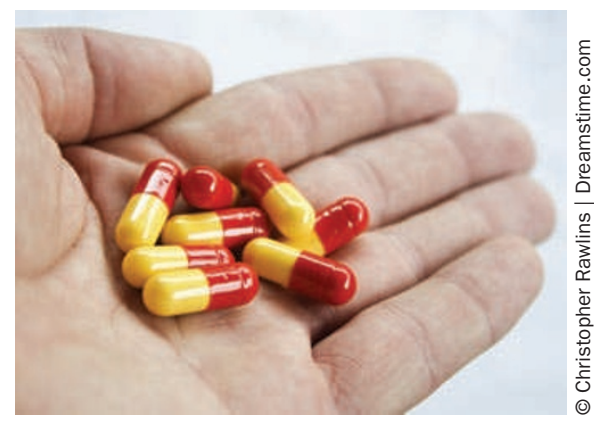

The results of this study highlight the importance of investigating the cause of VUR in children. "When faced with patients with persistent VUR, videourodynamics or noninvasive uroflowmetry and concomitant pelvic floor electromyogram monitoring are essential tools for evaluation of secondary causes of reflux, especially primary bladder neck dysfunction," says Kajbafzadeh.

The research team are now focusing on the short-term effects of prazosin on uroflowmetry parameters in children with VUR, as well as attempting to elucidate the cellular and tissue effects exerted by prazosin in the lower urinary tract.

Sarah Payton

Original article Kajbafzadeh, A. M. et al. Vesicoureteral reflux and primary bladder neck dysfunction in children: urodynamic evaluation and randomized, double-blind, clinical trial on effect of $\alpha$-blocker therapy. J. Urol. 184, 2128-2133 (2010) 